\title{
Synergistic Antitumor Activity of Camptothecin-Doxorubicin Combinations and their Conjugates with Hyaluronic Acid
}

Kathryn M. Camacho, Sunny Kumar, Stefano Menegatti, Douglas R. Vogus, Aaron C. Anselmo, and Samir Mitragotri*

Department of Chemical Engineering

University of California at Santa Barbara

Santa Barbara, CA, 93106

* Send correspondence to Prof. Samir Mitragotri: samir@engineering.ucsb.edu, Ph: 805-698-6196, Fax: 805-8934731 


\begin{abstract}
Combinations of topoisomerase inhibitors I and II have been found to synergistically inhibit cancer cell growth in vitro, yet clinical studies of these types of combinations have not progressed beyond phase II trials. The results of clinical combinations of topoisomerase (top) I and II inhibitors typically fall within one of two categories: little to no improvement in therapeutic efficacy, or augmented toxicity compared to the single drug counterparts. Hence, despite the promising activity of top I and II inhibitor combinations in vitro, their clinical applicability has not been realized. Here, we report the use of polymer-drug conjugates as a means to co-deliver synergistic doses of top I and II inhibitors camptothecin (CPT) and doxorubicin (DOX) to tumors in vivo in a 4T1 breast cancer model. At specific molar ratios, DOX and CPT were found to be among the most synergistic combinations reported to date, with combination indices between 0.01 and 0.1 . The identified optimal ratios were controllably conjugated to hyaluronic acid, and elicited significant tumor reduction of murine 4T1 breast cancer model when administered intravenously. This study elucidates a method to identify synergistic drug combinations and translate them to in vivo by preserving the synergistic ratio via conjugation to a carrier polymer, thus opening a promising approach to translate drug combinations to clinically viable treatment regimens.
\end{abstract}

Keywords: chemotherapy, drug combinations, polymer drug conjugate, synergistic 


\section{Introduction}

Since the 1960s, combinations of chemotherapy agents have been projected to eminently alter cancer therapies by taking after the successful model of tuberculosis treatments and addressing drug-resistant strains $[1,2]$. To that end, numerous efforts have focused on identifying potent anticancer combinations with top I and II inhibitors serving as one of the most widely studied classes [3-17]. These immense efforts are attributed to their collateral drug sensitivity; top Iexposed cancer cells compensate the obstruction of DNA replication by enhancing top II activity, which further sensitizes the cancer cells to top II inhibitors [18, 19]. Although top I and II inhibitors have been found to synergistically inhibit cancer cell growth in vitro [20-24], their clinical applicability has been questionable due to little improvements in tumor reduction $[6,13$, 17] and heightened side effects [4, 5, 12, 13]. For example, archetypal top II inhibitor doxorubicin (DOX) can achieve $>20 \%$ overall response (patients with reduced tumor sizes) in small cell lung cancer [25], but when administered with top I inhibitor irinotecan, the combination showed no improvement in treatment efficacy and only resulted in $12.9 \%$ overall response (OR) in phase II trials [17]. Other top I and II inhibitor combinations, such as amrubicin and irinotecan, show improved OR (up to 67\%), but severe hematological toxicity in $71 \%$ of patients [5]. One clinical trial, consisting of the combination of topotecan and pegylated liposomal DOX, was even forced to terminate due to dose-limiting toxicity; the result was the inability to identify a tolerable combination dose [13]. Thus, although top I and II inhibitors synergistically inhibit cancer cell growth in vitro, their clinical translation has been stagnated by poor therapeutic efficacies and dose-limiting toxicities. The study reported here demonstrates the use of polymer-drug conjugates as a means to overcome these limitations and translate top I and II inhibitor potency in vivo by simultaneously delivering synergistic ratios of the drugs to tumors.

Conjugation to polymers can help achieve clinical efficacy of top I and II inhibitors by promoting drug accumulation in tumors rather than essential organs via the enhanced permeation and retention (EPR) effect [26-29], and by ensuring that the tumors are exposed to synergistic concentrations of the drug combination. Polymer-drug conjugates are actively explored for the administration of single chemotherapy agents and have already shown clinical benefits over free drug injections [30]: reduced liver accumulation, enhanced drug localization in tumors, and improved drug circulation times [31]. Furthermore, since multi-drug incorporation is governed by chemical reactions as opposed to traditional hydrophobic encapsulations, precise ratios of the drugs can be conjugated to the polymer and delivered to tumor tissue. This characteristic of polymer-drug conjugates is crucial since drug ratios can govern whether the combination is synergistic or antagonistic [32, 33].

Here, we identify a synergistic combination of a model top I (camptothecin (CPT)) and top II inhibitor (DOX), and report a means to deliver the combination in vivo through conjugation to a natural water-soluble biopolymer hyaluronic acid (HA). HA was chosen as the 
polymer carrier, not just for its biocompatibility, but also for its specificity for surface marker CD44, which is over-expressed on many cancer cells [34-36].

\section{Materials and Methods}

\section{Materials}

Camptothecin (CPT), N-(3-Dimethylaminopropyl)-N'-ethylcarbodiimide hydrochloride (EDC), 4-(dimethylamino)pyridine (DMAP), ethylenediamine, Tween-80 and rhodamine B (RhoB) were purchased from Sigma-Aldrich (St. Louis, MO, USA). Doxorubicin (DOX) was obtained from LC Laboratories (Woburn, MA, USA). Alexa Fluor 647 Annexin V (Annexin V-647) was purchased from BioLegend (San Diego, CA, USA), and Sytox Green was obtained from Life Technologies (Grand Island, NY, USA). Hyaluronic acid (HA) of $250 \mathrm{kDa}$ MW was purchased from Creative PEGWorks (Winston Salem, NC, USA). 3-(4,5-Dimethylthiazol-2-yl)-2,5diphenyltetrazolium bromide (MTT), DRAQ5, and Hoechst were purchased from Invitrogen Life Technologies. Breast cancer HER2-overexpressing cell line BT-474, mouse metastatic breast cancer cell line 4T1, mouse brain endothelial cell line bEnd.3, Hybri-Care medium, Dulbecco's Modified Eagle's medium (DMEM) and cell culture grade water were acquired from ATCC. Fetal bovine serum (FBS), phosphate buffered saline (PBS), RPMI-1640 media, 2-(Nmorpholino)ethanesulfonic acid (MES) buffer, $0.25 \%$ trypsin, penicillin/streptomycin, and Nunc Lab-Tek 8-chambered coverglasses were purchased from Thermo Scientific. Cell culture flasks and microplates were purchased from Corning (NY, USA). Sephadex G-25 PD-10 columns were obtained from GE Healthcare Life Sciences (Piscataway, NJ, USA), and microcentrifuge filter tubes were purchased from EMD Millipore (Billerica, MA, USA). All other chemicals used for this study were obtained from Fisher Scientific and were the highest possible grade available.

\section{Cell Culture}

All cell lines were grown in a humidified incubator with $5 \% \mathrm{CO}_{2}$ at $37^{\circ} \mathrm{C}$. BT-474 cells were cultured in Hybri-Care medium supplemented with $10 \% \mathrm{FBS}$, and 4T1 cells were cultured in RPMI-1640 medium supplemented with 10\% FBS and 1\% penicillin/streptomycin. Endothelial cell line bEnd.3 was cultivated in DMEM medium supplemented with $10 \%$ FBS and $1 \%$ penicillin/streptomycin.

\section{Cell Viability and Drug Combination Studies}

BT-474 or bEnd. 3 cells were seeded in a 96-well cell culture plate at a density of 10,000 cells per well in a total volume of $100 \mu \mathrm{L} /$ well and allowed to adhere overnight. Media was then replaced with fresh media containing $\operatorname{drug}(\mathrm{s})$. Drugs were first dissolved in DMSO, and then further 
diluted in full cell culture medium so as not to expose cells to more than 0.5 vol\% DMSO. BT474 cells were incubated with drug solutions for 72 hours, while bEnd.3 cells were exposed to drug solutions for 48 hours. Drug incubation times were chosen such that control cells were $70 \%$ confluent after incubation. To assess cell viability, the media was replaced with $0.5 \mathrm{mg} / \mathrm{mL}$ MTT in media and allowed to incubate for 4 hours. Media was aspirated and replaced with dimethyl sulfoxide (DMSO) to solubilize intracellularly reduced MTT (formazan crystals). Formazan dye intensity was determined by absorbance measured at $570 \mathrm{~nm}$ in a Tecan Infinite M200 Pro plate reader (Männedorf, Switzerland). The fraction of inhibited cell growth, also known as fractional cell inhibition, was calculated by subtracting live cells in experimental wells from untreated control cells and normalizing against control cells. $\mathrm{D}_{50}$ refers to the concentration of drug required to inhibit $50 \%$ cell growth.

Experimental cytotoxicity data were fitted to the median-effect model developed by Chou and Talalay to obtain in vitro cytotoxicity curves for each drug [37]. Drugs were combined in a variety of molar ratios (R) and assessed for synergy utilizing Chou and Talalay's Combination Index (CI). Synergism, additivism, and antagonism were indicated by CI values less than 1, equal to 1 , and greater than 1 , respectively.

\section{Annexin V/Sytox Green Apoptosis Assay}

Apoptosis assessment was made based on Annexin V and Sytox Green counterstaining. Studies followed the Life Technologies Apoptosis Assay protocol, with few modifications tailored to BT-474 cells. Briefly, cells were seeded at a concentration of $100 \times 10^{4}$ cells per $25 \mathrm{~cm}^{2}$ cell culture flask in a total volume of $10 \mathrm{~mL}$ media, and allowed to adhere overnight. Cells were exposed to drug solutions for 72 hours, as previously described. After drug exposure, adherent and floating cells were harvested at a concentration of $1 \times 10^{6}$ cells $/ \mathrm{mL}$ in Annexin $\mathrm{V}$ binding buffer, and $200 \mu \mathrm{L}$ of each sample were incubated with $5 \mu \mathrm{L}$ of Annexin V-647 and $1 \mu \mathrm{L}$ of 1 $\mu \mathrm{M}$ Sytox Green. After 15 minutes of dye incubation, cells were diluted 5X in ice cold Annexin $\mathrm{V}$ Binding Buffer, and immediately analyzed via flow cytometry in a Becton Dickinson FACSAria cell sorter (Franklin Lakes, NJ, USA). To quantify Annexin V-647 and Sytox Green fluorescence, a $633 \mathrm{~nm}$ laser with 660 PMT and a $488 \mathrm{~nm}$ laser with 530 PMT, respectively, were utilized. Cells gated as -AV/-SG were live, cells with +AV/-SG were pre-apoptotic, and cells gated as $+\mathrm{AV} /+\mathrm{SG}$ were either late apoptotic or necrotic.

\section{Synthesis of CPT-HA, DOX-HA, and CPT-HA-DOX}

CPT and DOX were conjugated to HA via nucleophilic acyl substitution (Fig. S1). Specifically, the carboxylic acid moieties of HA were conjugated to CPT via ester formation [38] and to DOX

via both ester formation and aminolysis [39]. For all reactions, $100 \mathrm{mg}$ of $250 \mathrm{kDa}$ MW HA was 
dissolved in a $10 \mathrm{~mL}$ mixture of DMSO/water (1:1 by volume) under stirring and slight heating $\left(40^{\circ} \mathrm{C}\right)$. DMAP and EDC were added at a molar ratio of 0.75:1 relative to HA monomers, and were allowed to activate the polymer for 1 hour under stirring. To synthesize CPT-conjugated HA (CPT-HA), CPT was slowly added to the reaction mixture at a molar ratio of 0.4:1 CPT:HA. In the case of DOX-conjugated HA (DOX-HA), DOX was dissolved in the reaction mixture in a molar ratio of 0.2:1 DOX:HA. To synthesize CPT- and DOX-conjugated HA (CPT-HA-DOX), CPT was first conjugated to HA for 3 days, followed by the subsequent reaction of DOX to CPTHA. The reactions proceeded under slight heating $\left(40^{\circ} \mathrm{C}\right)$ for 3 days. Afterwards, CPT-HA and DOX-HA were separated from unreacted free drugs, EDC and DMAP via size exclusion chromatography through Sephadex G-25 PD-10 desalting columns (5000 MW exclusion limit) equilibrated in phosphate buffered saline (PBS, $\mathrm{pH} 7.4)$. The reaction products were further concentrated in $0.5 \mathrm{~mL}$ centrifugal filter tubes (3000 NMWL) for a minimum of three runs, each at $16000 \mathrm{~g}$ for $15 \mathrm{~min}$. For the final centrifuge run, an additional $0.4 \mathrm{~mL}$ of PBS was added. Concentrations of CPT and DOX were determined via absorbance at $366 \mathrm{~nm}$ and $480 \mathrm{~nm}$, respectively, of formulation serial dilutions utilizing a Tecan Infinite M200 Pro plate reader. In both CPT-HA and DOX-HA formulations, $1.6 \mathrm{~mol} \%$ drug:HA was achieved. The drug encapsulation in CPT-HA-DOX was $5.9 \mathrm{~mol} \% \mathrm{CPT}$ and $1.8 \mathrm{~mol} \%$ DOX.

\section{Release of CPT or DOX from HA Conjugates}

Drug release of CPT or DOX from CPT-HA or DOX-HA conjugates was achieved utilizing Slide-A-Lyzer MINI Dialysis Devices of 10,000 MWCO (Life Technologies, Grand Island, NY).

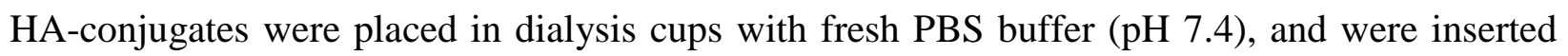
into microcentrifuge tubes containing $1 \mathrm{~mL}$ PBS. Dialysis devices were placed at $37^{\circ} \mathrm{C}$ and allowed to shake at 100 RPM. At indicated time points, concentrations of CPT or DOX in the medium surrounding the dialysis cup were determined via absorbance. External PBS buffer was replenished at each time point. Drug release was fit to exponential release profiles in order to estimate time required for half of the drug to release, indicated by $t_{1 / 2}$.

\section{CPT-HA or DOX-HA Internalization}

To assess the effect of HA-conjugation on the internalization of CPT or DOX in BT-474 cells, confocal laser scanning microscopy was utilized. Cells were seeded at a density of 80,000 cells per $300 \mu \mathrm{L}$ media in an 8-well chambered borosilicate coverglass (Nunc Lab-Tek), and were allowed to adhere overnight. Cells were exposed to the same drug concentration, the free drug $\mathrm{D}_{50}$, of either the free drug solution or the HA-conjugate form for $24 \mathrm{hrs}$ at $37^{\circ} \mathrm{C}$ and $5 \% \mathrm{CO}_{2}$. Following drug incubation, cells were washed twice with warmed PBS (pH 7.4), followed by staining with nuclear dyes at $37^{\circ} \mathrm{C}, 5 \% \mathrm{CO}_{2}$. In the case of DOX-exposed cells, Hoechst nuclear dye was utilized at a concentration of $25 \mu \mathrm{g} / \mathrm{mL}$ for 30 minutes. For CPT-exposed cells, to avoid overlap fluorescence between CPT and nuclear marker, DRAQ5 was used to stain nuclei at a concentration of $5 \mu \mathrm{M}$ in media for 60 minutes. Cells were washed twice more in warmed PBS and finally suspended in media immediately prior to imaging. All cells were imaged live with an 
Olympus Fluoview 1000 spectral confocal equipped with a $60 \mathrm{X}$ silicon oil objective and a $37^{\circ} \mathrm{C}$ temperature-controlled imaging chamber. DOX, CPT, Hoechst and DAPI were excited with the following lasers: $488 \mathrm{~nm} 10 \mathrm{~mW}$ Argon gas (DOX), $405 \mathrm{~nm} 50 \mathrm{~mW}$ diode (CPT or Hoechst), and $635 \mathrm{~nm} 20 \mathrm{~mW}$ diode (DRAQ5). Z-stacks of $10 \mu \mathrm{m}$ were captured and subsequently analyzed with ImageJ $1.47 \mathrm{~h}$ software (NIH). Each $\mathrm{z}$-stack was collapsed into an averaged image, and fluorescence intensity was reported as the raw integrated density divided by number of cells. An average of 25 cells was imaged in each field view.

HA internalization studies were conducted similarly. HA-Fluorescein (HA-F) of 250kDa MW was purchased from Creative PEGWorks and directly dissolved in PBS, pH 7.4. The polymer was further diluted in media, and then incubated with BT-474 cells for 6 hours. To evaluate the need for energy-intensive internalization, cells were incubated at either $37^{\circ} \mathrm{C}$ or $4^{\circ} \mathrm{C}$. Hoechst was used to label cell nuclei. HA-F was excited at $488 \mathrm{~nm}$, and analysis was performed as described above.

\section{In Vivo Efficacy and Toxicity}

A mouse 4T1 breast tumor model was utilized to evaluate in vivo tumor reduction by CPT + DOX formulations. All experiments were performed according to approved protocols by the Institutional Animal Care and Use Committee of the University of California, Santa Barbara. Six to eight week old female BALB/c mice were purchased from Charles River Laboratories (Wilmington, MA, USA). Tumor inoculation was achieved by subcutaneously injecting $5 \times 10^{4}$ 4T1 cells in the abdominal mammary gland in order to prevent interference with normal bodily functions. Prior to injection, 4T1 cells were washed twice in PBS and re-suspended in sterile saline $(0.9 \mathrm{wt} / \mathrm{vol} \% \mathrm{NaCl})$. Mice were randomly assigned to experimental and control groups, and were treated every other day with specified formulations beginning on day 3 postinoculation, for a total of five treatments (Days 3, 5, 7, 9, 11). Mice were treated with i.v. tail injections of either CPT-HA-DOX or free CPT + DOX in equivalent drug doses of $2 \mathrm{mg} / \mathrm{kg} \mathrm{CPT}$ and $1.05 \mathrm{mg} / \mathrm{kg}$ DOX. To prepare the free drug formulation, CPT was first dissolved in $10 \mathrm{vol} \%$ Tween-80 in $0.9 \mathrm{wt} / \mathrm{vol} \% \mathrm{NaCl}$. DOX was directly dissolved in $0.9 \mathrm{wt} / \mathrm{vol} \% \mathrm{NaCl}$, and was subsequently mixed with the CPT solution. CPT-HA-DOX was freshly prepared in $0.9 \% \mathrm{NaCl}$ prior to injections. Tumor growth inhibition was assessed with tumor volume, quantified by $V=$ $1 / 2(l) x(w)^{2}$, where $l$ is the longest tumor diameter and $w$ is the shortest tumor diameter measured by a digital caliper. Caspase-3 immunohistochemistry and hematoxylin and eosin (H\&E) histology analyses were also performed on excised tumors post-treatment in order to further assess tumor growth inhibition. Tumors were fixed and further sent to Mass Histology Services, Inc., where the tissues were paraffin-blocked, stained, and analyzed. To evaluate formulation toxicity, body weights were measured daily. Additionally, liver, spleen, heart, and lung organs were harvested at the end of the experiments, fixed and further analyzed by Mass Histology Services, Inc. (Worcester, MA, USA) via H\&E staining.

Statistical Analysis 
Statistical significance was assessed via the two-tailed, unpaired Student's $t$-test in Microsoft Excel. One star indicates significance with $\mathrm{p}<0.05$, and two starts indicate $\mathrm{p}<0.01$.

\section{Results}

Synergistic activity of CPT and DOX in human breast cancer cells

CPT and DOX were tested for synergistic cell growth inhibition of human breast cancer cell line BT-474 after 72 hour exposure (Fig. 1a). Synergy was quantified as the Combination Index (CI) [37]; CI values lower than 1 indicate synergy whereas values greater than 1 imply antagonism. $\mathrm{CI}$ decreased with increasing molar ratio $(\mathrm{R})$ of drugs, with the greatest synergy occurring when $\mathrm{R}(\mathrm{CPT}: \mathrm{DOX})>2(0.01<\mathrm{CI}<0.08)$. The highest CI was $1 \pm 0.07$, indicating that CPT and DOX interactions were never worse than additive. These favorable drug interactions are congruent with literature reports on synergy between topoisomerase I and topoisomerase II inhibitors [18-23]. CPT and DOX were also exposed to brain endothelial cell line bEnd.3 as a model healthy cell line (Fig. 1c), and were found to exhibit extreme antagonism (CI >>1) for all ratios investigated. The combination consistently inhibited less endothelial cell growth than CPT or DOX alone, suggesting that pair is considerably more toxic to cancer cells than endothelial cells.

Since CPT and DOX inhibit DNA top I and II, respectively, which are required to relieve DNA supercoiling during transcription and replication, exposure of either drug to cancer cells results in DNA damage and can induce apoptosis [40, 41]. Flow cytometry data indicated a significant increase in apoptosis when the drug pair was given in a synergistic ratio relative to treatment with DOX alone, and only little enhancement when provided in an additive ratio. Cells exposed only to CPT exhibited low percentages of apoptotic cells, incomparable to those treated with DOX alone, likely due to BT-474's inherent resistance to CPT. However, at a synergistic ratio $(\mathrm{R}=4.5, \mathrm{CI}=.05 \pm .01), \mathrm{CPT}+\mathrm{DOX}$ induced a $24 \%$ increase in early apoptotic population (Fig. 2), whereas cells exposed to additive ratio $(\mathrm{R}=0.1, \mathrm{CI}=1.00 \pm .07)$ resulted in only a $2 \%$ increase. 

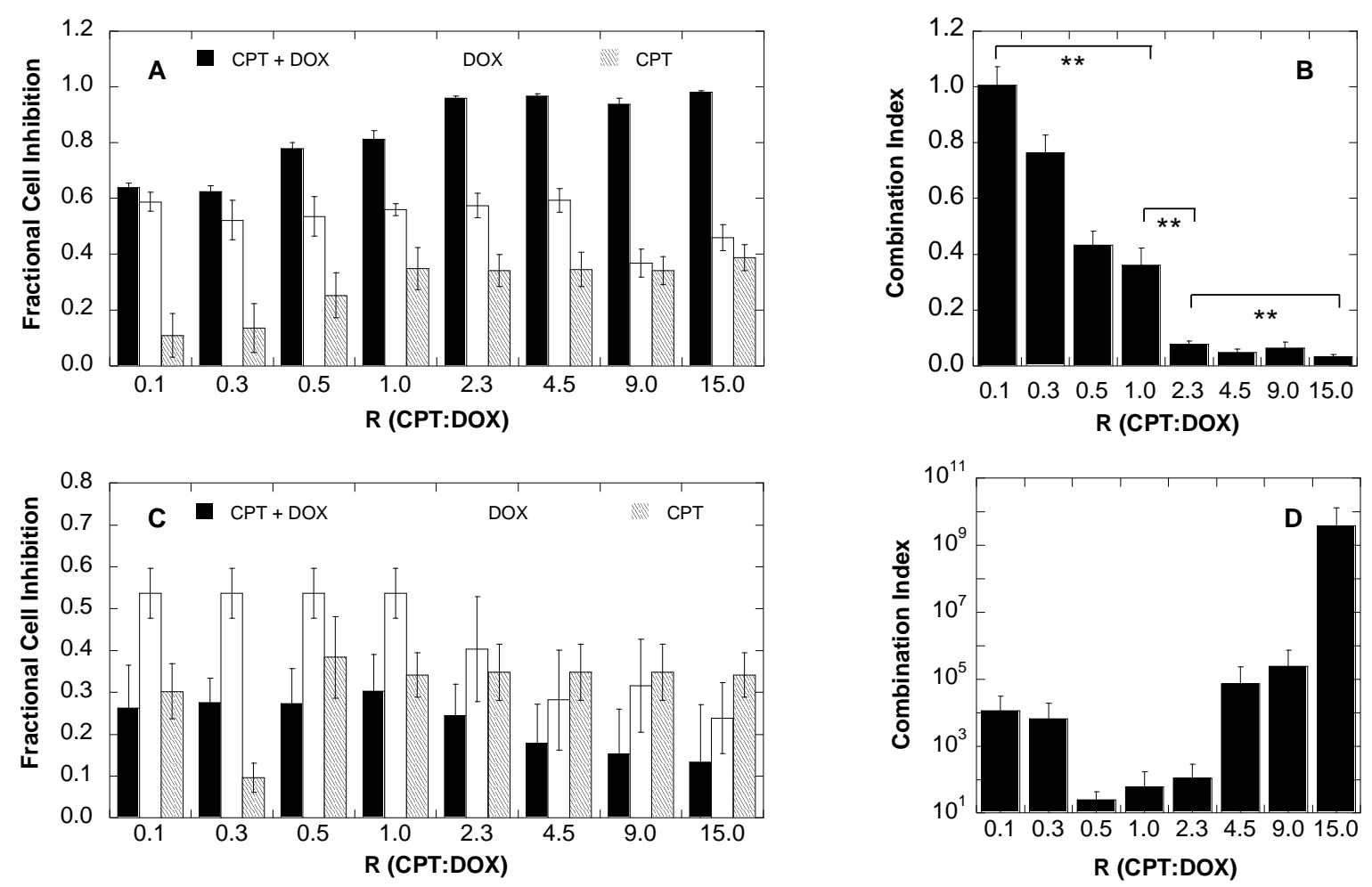

Fig. 1. The cell inhibition of human breast cancer cell line BT-474 (a,b) or mouse brain endothelial cell line bEnd.3 (c,d) treated with combinations of camptothecin (CPT) and doxorubicin (DOX) at various molar ratios (black bars). Single drug treatments of DOX (white) and CPT (hatched) at concentrations which make-up the combination are juxtaposed for direct comparison. Cells were assessed for viability post-drug incubation via MTT cytotoxicity assays. In the case of BT-474 treatments, drug concentrations $(\mu M)$ of CPT and DOX respectively corresponding to each $R$ ratio were: $0.1(0.05,0.5), 0.3(0.1$, 0.36), $0.5(0.2,0.4), 1.0(0.4,0.4), 2.3(0.9,0.4), 4.5(1.35,0.3), 9.0(2.0,0.22), 15.0(5.0,0.33)$. CPT and DOX concentrations $(\mu \mathrm{M})$ respectively utilized for bEnd.3 studies corresponding to each $R$ ratio were: $0.1(0.04,0.4), 0.3(0.11,0.4), 0.5(0.2,0.4), 1(0.4,0.4), 2.3(0.45,0.2)$, $4.5(0.45,0.1), 9(0.45,0.05), 15(0.3,0.02)$. Drug concentrations were chosen such that at least one of the individual drug doses inhibited between $40-60 \%$ cell growth. Combination Index (CI) values were calculated for the CPT and DOX combinations for (b) BT-474 and (d) bEnd.3 cells. Data expressed as mean \pm SD $(n=6)$. ** $p<0.01$. 

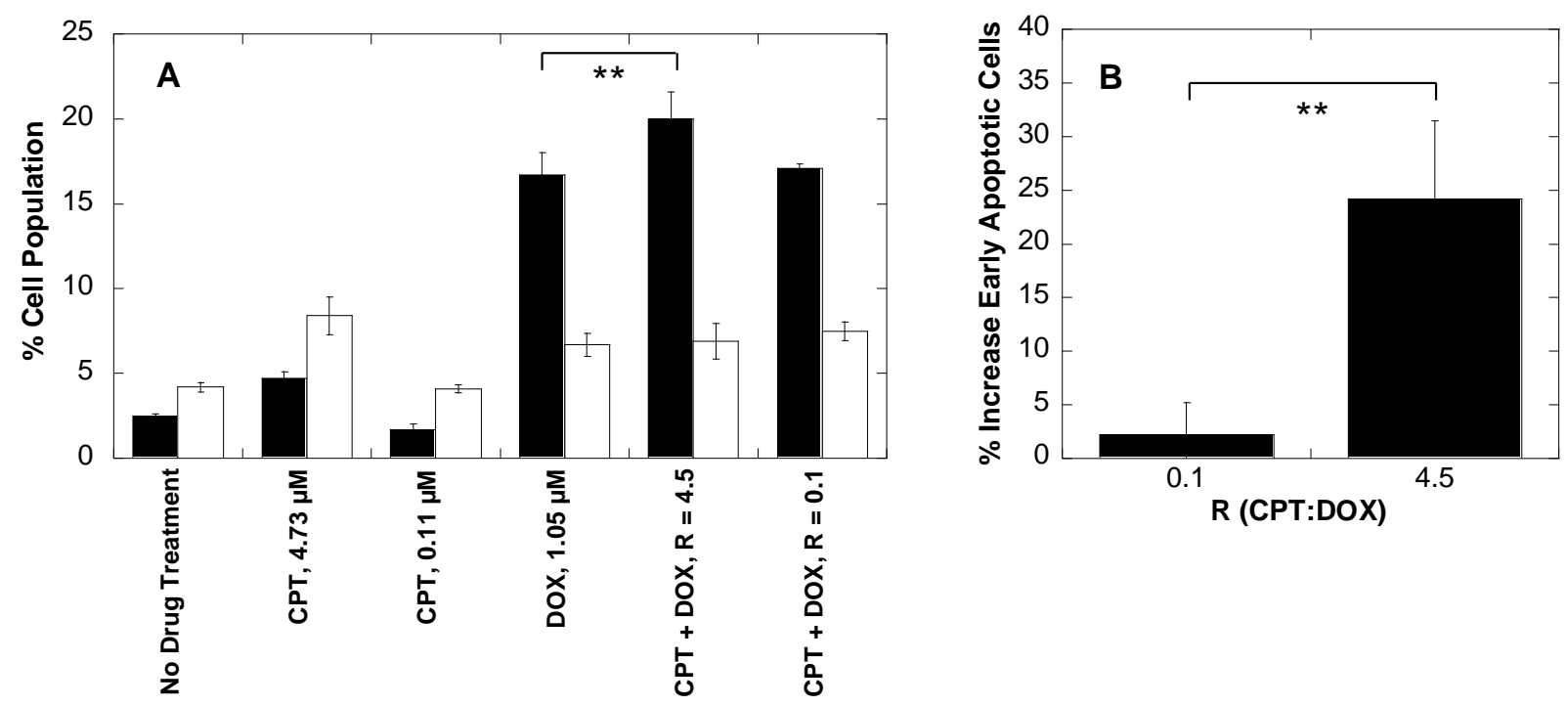

Fig. 2. Annexin V/Sytox Green assay was utilized to detect percentage of early apoptotic cells in various drug-treated BT-474 cells. Cells were treated with the following drugs for 72 hours prior to staining and analysis via flow cytometry: no drug treatment, $4.73 \mu \mathrm{M}$ CPT, $0.11 \mu \mathrm{M}$ CPT, $1.05 \mu \mathrm{M}$ DOX, $4.73 \mu \mathrm{M}$ CPT $+1.05 \mu \mathrm{M}$ DOX $(R=4.5$, representative synergistic dose), $0.11 \mu \mathrm{M}$ CPT $+1.05 \mu \mathrm{M}$ DOX $(R=0.1$, representative additive dose). (a) Cell populations were quantified via flow cytometry as follows: early apoptotic (black bars), and necrotic and/or late apoptotic (white bars). See Fig. S2 for corresponding scatter plots. (b) Percent enhancement of early apoptotic cells relative to treatment with DOX alone. Data represents mean \pm SD $(n=3)$. ** $p<0.01$.

Conjugation of CPT or DOX to HA

CPT and DOX were conjugated to HA so as to preserve their molar ratio after delivery into the body. Conjugation to HA reduced the activity of DOX compared to free drug (Fig. 3a), whereas the activity of CPT-HA conjugate was reduced only slightly compared to that of free CPT (Fig. $3 b)$. 

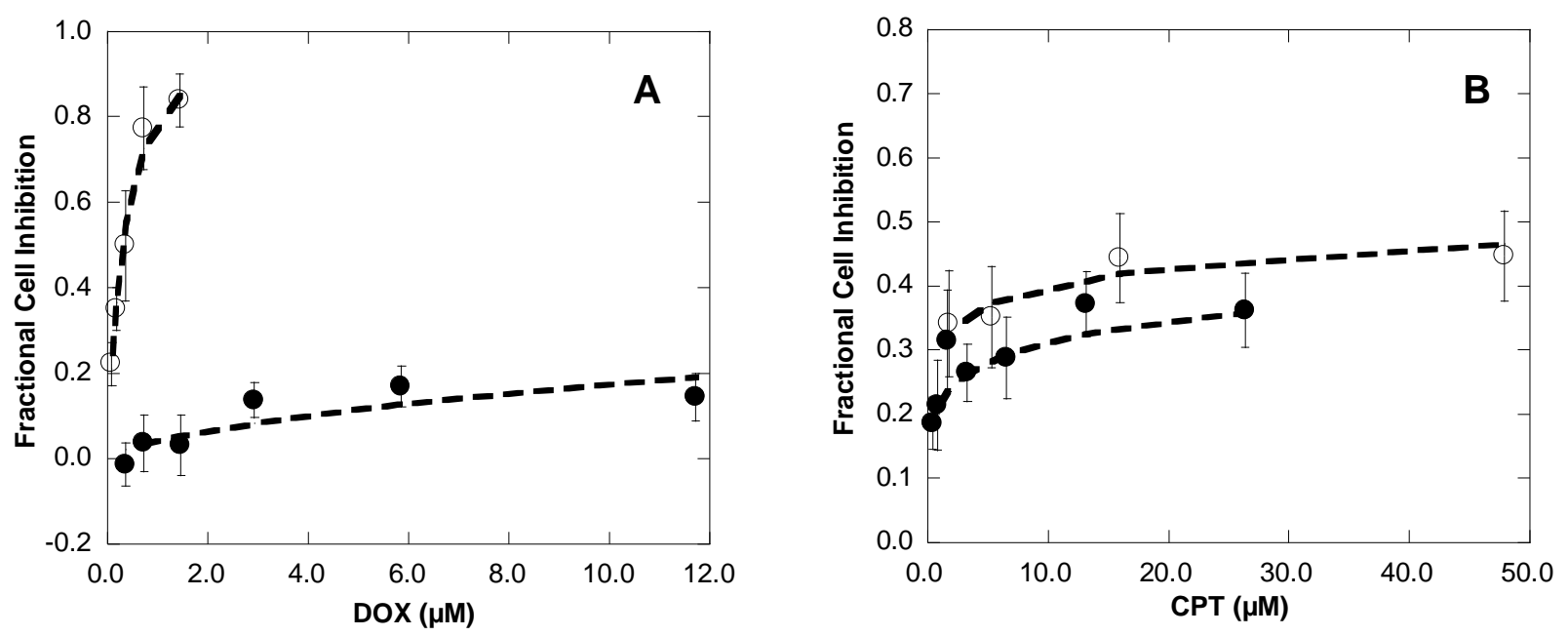

Fig. 3. Cell inhibition of human breast cancer cell line BT-474 in the presence of (a) DOX in free solution (open circles) or DOX-HA (filled circles) and (b) CPT in free solution (open circles) or CPT-HA (closed circles). Cells were incubated with each drug for 72 hrs, then analyzed via MTT assay. Data expressed as mean \pm SD ( $\geq 8)$. Dashed lines represent doseeffect curves fit to experimental data using Chou and Talalay's median-effect model.

Internalization and release studies were performed to investigate the origins of shifts in drug activity upon HA conjugation. HA was internalized by an active process as confirmed by lack of internalization at $4^{\circ} \mathrm{C}$ (Fig. S3). CPT-HA exhibited significantly enhanced uptake compared to free CPT (Fig. 4a) possibly due to its enhanced water solubility upon conjugation to HA. Uptake of DOX-HA on the other hand was only slightly less than that of free DOX (Fig. 4a). Subsequent intracellular distribution of HA-DOX, however, was significantly different compared to that of free DOX (Fig. 4c). HA-bound DOX was localized mostly in punctate spots surrounding nuclei, whereas free DOX was more diffuse and co-localized with cell nuclei. Whereas free DOX is sufficiently small to permeate across the cell and nuclear membrane, HADOX is likely internalized via endocytosis and localized in endosomes. Once internalized, DOX must release from the polymer via amide or ester hydrolysis and escape from the endosomes before reaching its nuclear target, top II. Drug release profiles (Fig. 5) indicate slow DOX release surmounting to only $25 \%$ after 72 hours, and a fast CPT release with near complete hydrolysis within 24 hours. The difference in drug release rates may be attributed to greater stability of amide bonds compared to ester bonds in aqueous conditions. This alternate internalization pathway, slow drug release, and reduced concentrations of free intracellular DOX may cause a time-lag between DOX internalization and activity, and may explain reduced cytotoxicity upon HA-conjugation. An exponential fit to the DOX release profile reveals a halflife of 254 hours, and suggests that complete DOX release will be achieved on the order of 50100 days. Therefore, in order to realize the full therapeutic potential of DOX-HA, drug incubations for longer time-periods than are reasonably utilized may be warranted. HA-bound CPT, on the other hand, improved intracellular CPT concentrations with no hydrolysis limitations; thus CPT-HA was only slightly less active compared to free CPT. 

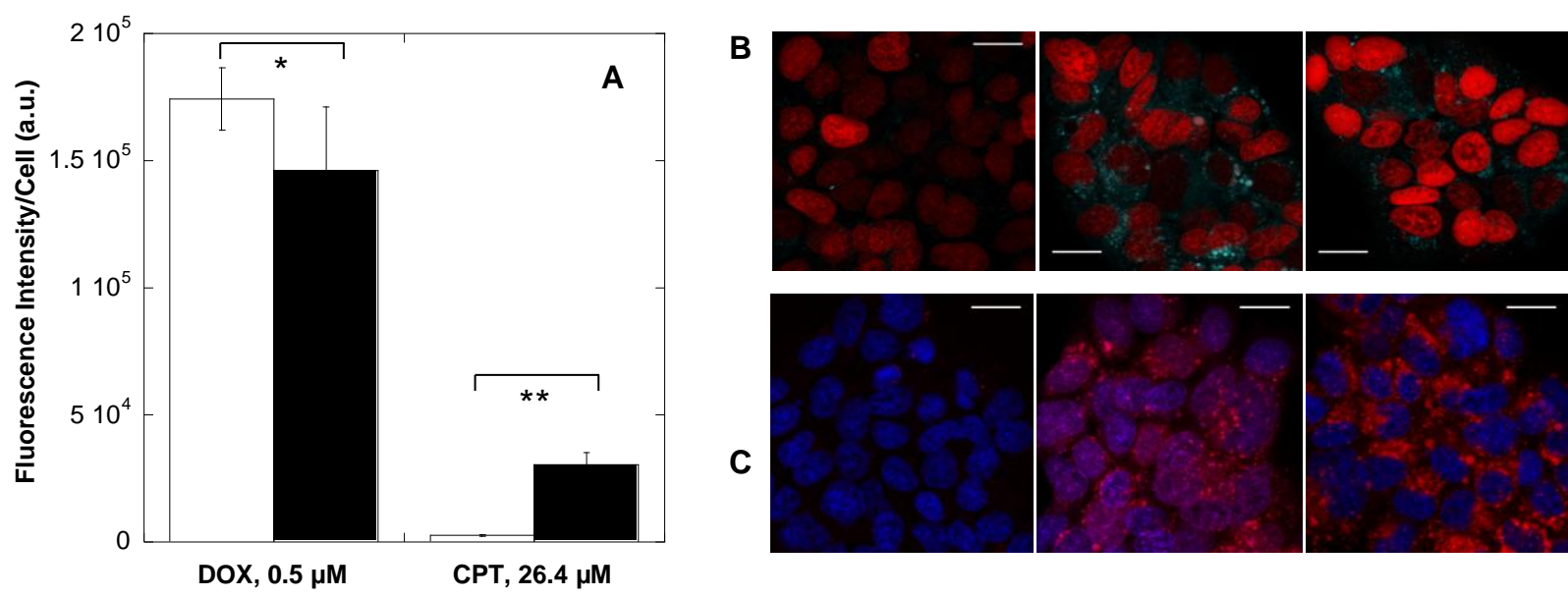

Fig. 4. (a) Quantitative fluorescence of DOX or CPT in free solution (white bars) or as a HA-conjugate form (black bars) present in BT-474 cells after 24 hours of incubation at $37^{\circ} \mathrm{C}$ and $5 \% \mathrm{CO}_{2}$. Data expressed as mean $\pm \mathrm{SD}(\mathrm{n}=3)$. (b) Representative images of CPT (cyan) internalization when incubated as a free solution (middle) or as a HA-conjugate (right). Untreated cells are demonstrated on the left. Cells were labeled with DRAQ5 nuclear dye (red). (c) Representative images of DOX (red) internalization when incubated as a free solution (middle) or as a HA-conjugate (right). Untreated cells are on the left. Cells were labeled with Hoechst nuclear dye (blue). Images are averages of $10 \mu \mathrm{m}$ z-stacks. $* \mathbf{p}<0.05, * * \mathbf{p}<0.01$.

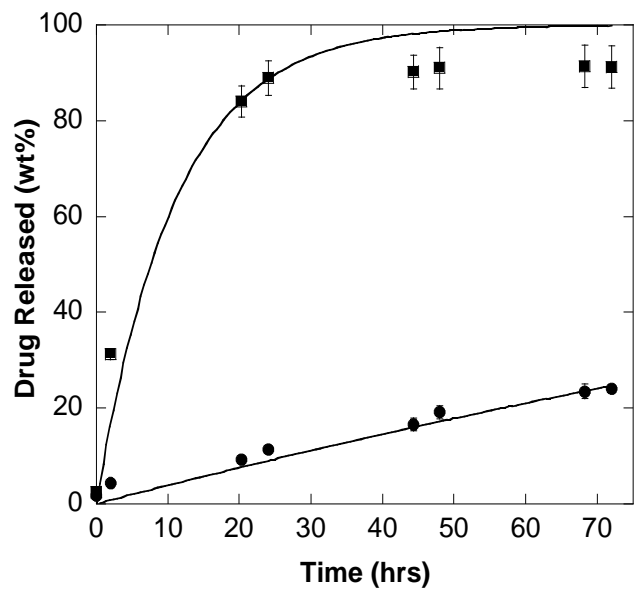

Fig. 5. Release of CPT (squares) or DOX (circles) from CPT-HA or DOX-HA, respectively, determined as wt\% of initial drug conjugated to HA. Lines represent exponential fits to release profiles $\left(t_{1 / 2, C P T}=11 \mathrm{hrs}, t_{1 / 2, \text { DOX }}=254 \mathrm{hrs}\right)$. Data represents mean $\pm S D(n=3)$. 
CPT-HA and DOX-HA were mixed at various molar ratios and incubated with BT-474 cells, followed by CI analysis. The combination inhibited more cancer cell growth than either CPTHA or DOX-HA alone (Fig. 6a). The CI of the mixed conjugates (Fig. 6b) was less than 1 for 1 $<\mathrm{R}<9$ CPT:DOX, indicating that CPT-HA and DOX-HA were indeed synergistic at the same ratios that free CPT and DOX elicited synergy (Fig. 1).

CPT and DOX were then co-conjugated to HA. For the purpose of demonstrating a synergistic drug-loaded carrier, HA carrying a molar ratio of $\mathrm{R}=3.2 \mathrm{CPT}$ :DOX was investigated. The content of CPT and DOX was determined via UV-Vis spectroscopy to be 5.9 mol\% and $1.8 \mathrm{~mol} \%$, respectively. In vitro cytotoxicity studies show that CPT-HA-DOX inhibited more cancer cell growth than either CPT-HA (Fig. 7a) or DOX-HA (Fig. 7b) alone. Cell inhibition studies performed with mixed drug HA-conjugates and drugs co-conjugated to HA reveal that polymer-conjugation preserved synergy between CPT and DOX. While both formulations conserved the drug pair's potency, only the latter can ensure simultaneous exposure to tumor cells, and can provide a means to capture the in vitro antitumor efficacy in vivo, as well.
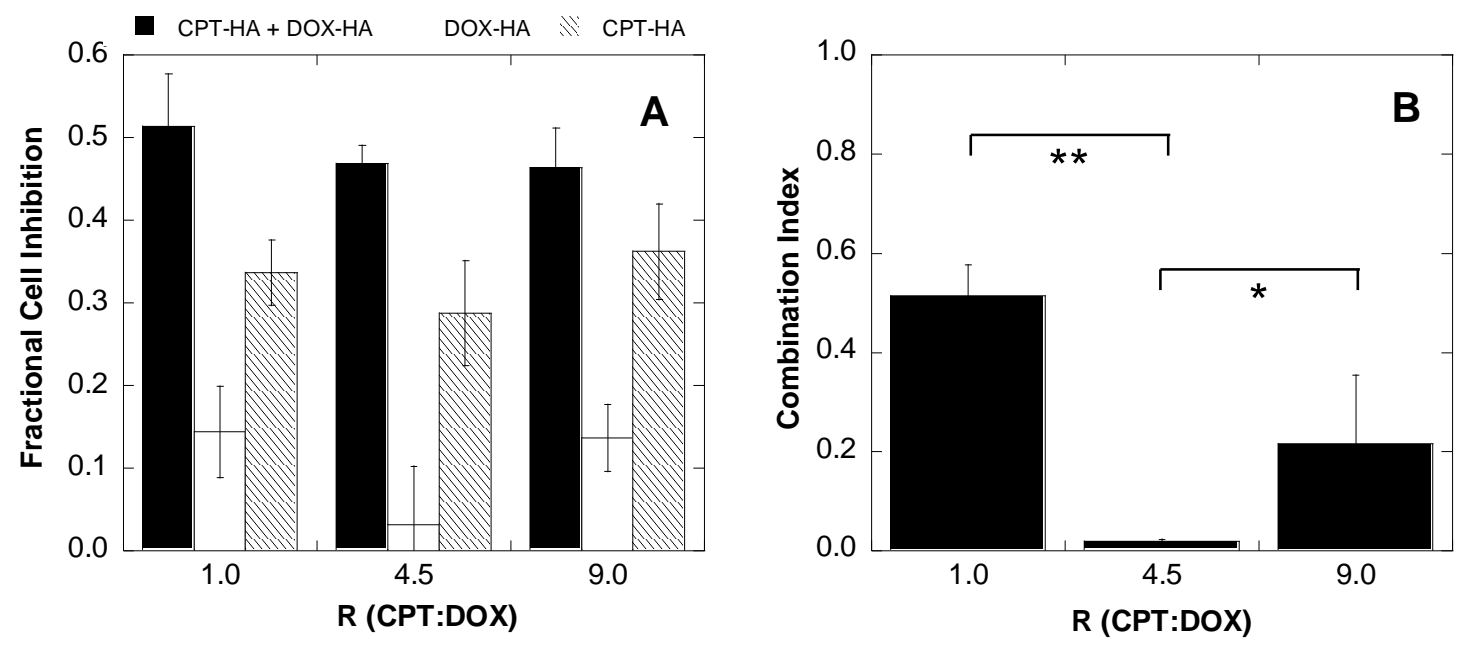

Fig. 6. (a) Cell inhibition of human breast cancer cell line BT-474 treated with combinations of CPT-HA and DOX-HA at various molar ratios (black bars). Single HAconjugate treatments of DOX-HA (white) and CPT-HA (hatched) at concentrations which make-up the combination are juxtaposed for direct comparison. Cells were incubated with HA-conjugates for 72 hours, and subsequently analyzed via MTT cytotoxicity assays. Data is represented as mean $\pm S D(n=6)$. Drug concentrations of CPT and DOX respectively in $\mu \mathrm{M}$ : 1 (11.7, 11.7), $4.5(6.6,1.5), 9(26.4,2.9)$, (b) Combination Index (CI) values calculated for the CPT-HA and DOX-HA combinations shown in Fig. 6(a). $* \mathrm{p}<0.05$, $* * \mathrm{p}<0.01$. 

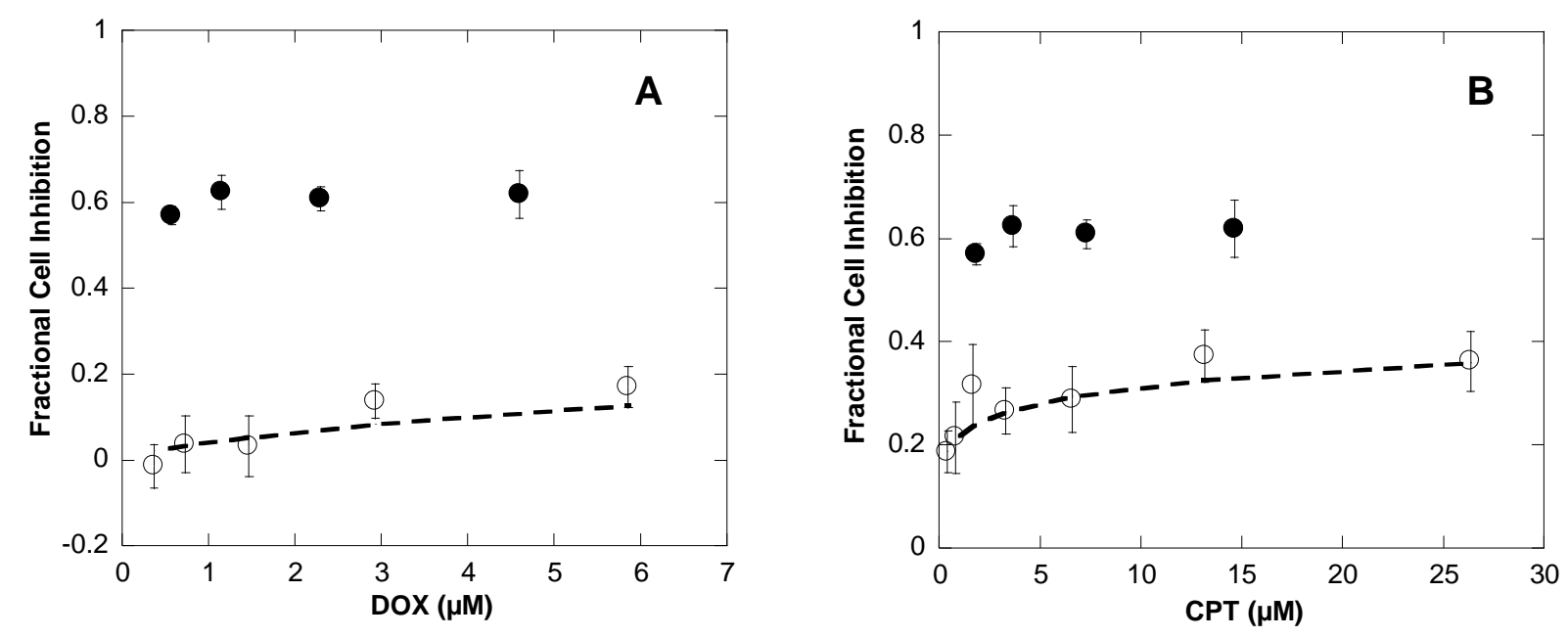

Fig. 7. (a) Cell inhibition of BT-474 cells in the presence of DOX-HA (open circles) or CPTHA-DOX conjugates (filled circles). (b) Comparison of the cell inhibition of CPT-HA (open circles) and CPT-HA-DOX conjugates of $R=3.2 \mathrm{CPT}$ :DOX (filled circles). Cells were incubated with formulations for $\mathbf{7 2}$ hours, and assessed for cell viability utilizing the MTT cytotoxicity assay. Data is reported as mean $\pm S D(n \geq 6)$.

In vivo anti-tumor efficacy

An orthotopic $4 \mathrm{~T} 1$ breast cancer mouse model in $\mathrm{BALB} / \mathrm{c}$ mice was utilized to assess the in vivo anticancer efficacy of CPT-HA-DOX. A 4T1 model was chosen for its robust tumor formation in mice [42], and to challenge the synergistic conjugate with a highly metastatic, aggressive cancer [43]. While all in vitro studies focused on the cell inhibition of BT-474 human breast cancer cells, CPT and DOX synergy was also verified in 4T1 cells and occurred at the same ratios as BT-474 (Figs. S4-S5). Mice were administered i.v. with designated formulations every other day for a total of 5 treatments. As high as $70 \%$ tumor volume reduction was achieved by the free drug combination (Fig. 8a), and 50\% tumor reduction was achieved with the coconjugated polymer relative to control. In both cases, negligible body weight change was observed (Fig. 8b). 

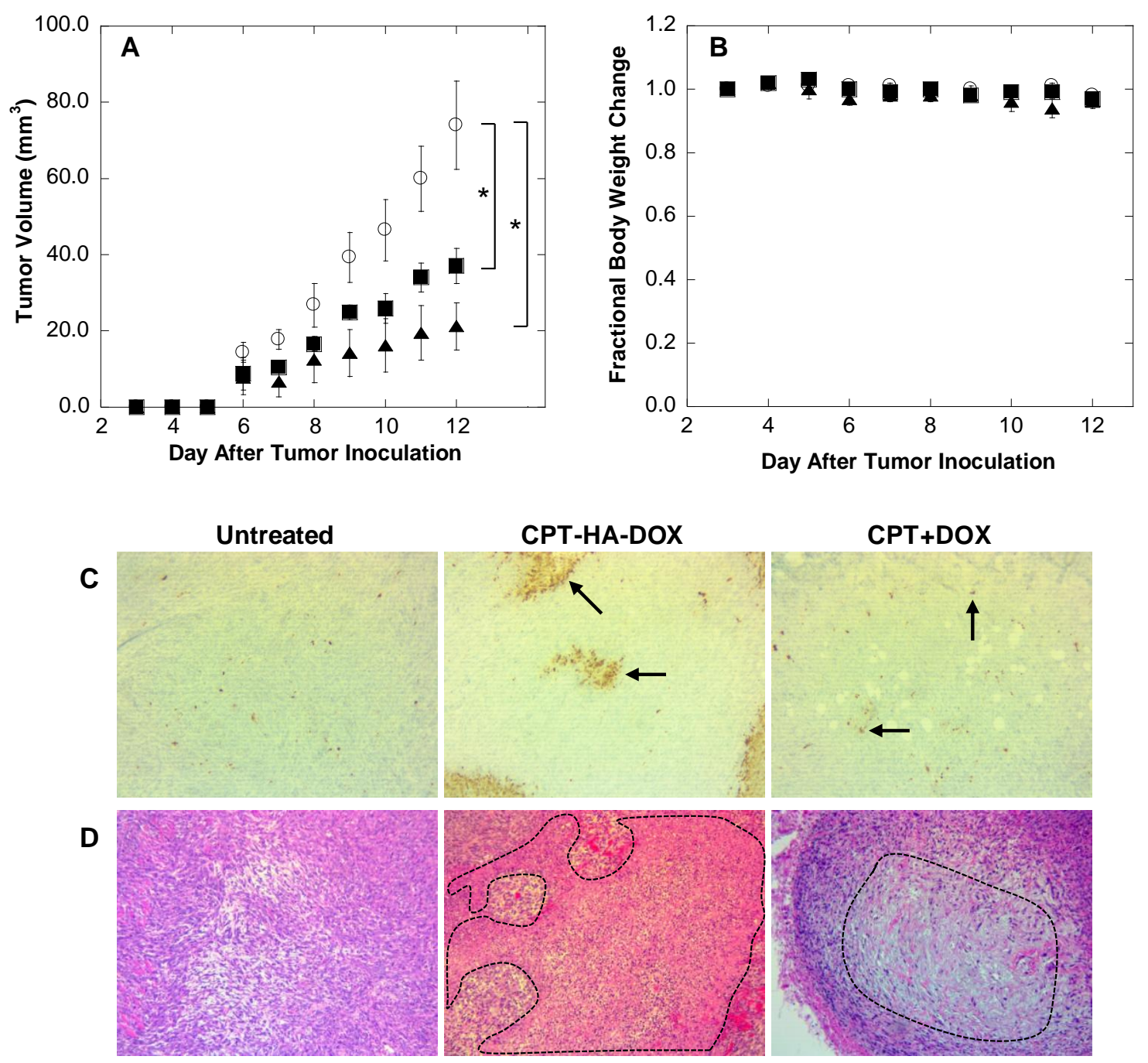

Fig. 8. (a) Tumor growth of mice bearing 4T1 breast cancer tumors, without treatment (open circles), with IV injections of CPT-HA-DOX (filled squares), or free CPT + DOX in $0.9 \%$ saline (filled triangles). BALB/c mice were inoculated with $5 \times 10^{4} 4 \mathrm{~T} 1$ breast cells in $40 \mu \mathrm{L}$ saline, and were injected i.v. every other day (beginning on day 3) with CPT-HADOX or free CPT + DOX, at a concentration of $1.05 \mathrm{mg} / \mathrm{kg}$ DOX and $2 \mathrm{mg} / \mathrm{kg}$ CPT for a total of 5 injections. Tumor volume was measured as $\left(l \times w^{2}\right) / 2$, where $l$ corresponds to the longest tumor diameter and $w$ corresponds to the shortest tumor diameter. (b) Change in body weight of control mice (open circles), CPT-HA-DOX-treated mice (filled squares), or CPT + DOX-treated mice (filled triangles). Data is reported as mean $\pm \mathrm{SE}$ ( $\mathrm{n} \geq 8$ ). (c) Immunohistochemistry analysis of excised control tumors (left), tumors treated with CPTHA-DOX (middle) or free CPT + DOX (right) stained for cleaved caspase-3 (apoptosis indicator). Arrows indicate representative stained apoptotic nuclei. (d) Histology analysis of tumors treated with H\&E. Circled areas represent necrotic tissue. Enlarged images for 


\section{clarification are provided in Fig. S6. All images were taken at 100X magnification and are representative of each group $(n=3)$. * $p<0.05$.}

Caspase 3 immunohistochemistry of tumor sections post-treatment was performed to further assess antitumor efficacy of CPT and DOX formulations. Despite greater gross tumor reduction in mice treated with free $\mathrm{CPT}+\mathrm{DOX}$, more apoptotic cells were found in CPT-HADOX-treated tumors (Fig. 8c). Furthermore, H\&E staining shows more necrosis in mice treated with CPT-HA-DOX compared to those treated with free CPT+DOX, as indicated by the greater occurrence of pyknotic (smaller) or missing nuclei (absence of purple staining) and overall pink rather than purple hue (Fig. 8d). Histology analyses also suggest that, while macroscopic measurements indicate that free $\mathrm{CPT}+\mathrm{DOX}$ mixture is more effective in reducing tumor size, CPT-HA-DOX was more effective in inducing both necrosis- and apoptosis-induced cell death. Histology analyses of essential organs post-treatment were conducted to further investigate formulation toxicity. Lung, spleen, and liver organs in both CPT + DOX-treated and CPT-HADOX-treated mice showed no toxicity (Fig S7). Heart sections resected from CPT-HA-DOXtreated mice indicated mild inflammation, whereas those from CPT + DOX-treated mice showed no toxicity. Overall, both formulations were well-tolerated with no severe side effects.

\section{Discussion}

Topoisomerase I and II inhibitors have a proven rationale, collateral drug sensitivity [18, 19], for favorable drug interactions against tumor cells, and have been shown to synergistically inhibit cancer cell growth in vitro [20-23], but when it comes to their clinical utility, escalation of adverse effects $[4,5,12,13]$ and little improvement in tumor reduction $[6,13,17]$ limit their use. This study demonstrates the use of HA as a means to deliver precise ratios of CPT and DOX for synergistic efficacy. Incorporation of synergistic CPT and DOX molar ratios onto HA was achieved through stoichiometric conjugations. The result was remarkable tumor reduction in vivo, and little toxicity in essential organs, likely due to the low chemotherapy doses required for therapeutic effect.

CPT and DOX were identified as highly synergistic with remarkably low CI values, and were thus chosen as a model combination for HA-conjugation. For molar ratios > 2 CPT:DOX, CI values were found to range between 0.01-0.1 (Fig. 1b). These values are among the lowest, and therefore most synergistic, of reported drug interaction studies evaluated by the CI method [24, 33, 44-48], rendering the pair highly desirable for co-delivery to tumors. Previous reports found CPT and DOX to be only additive in wild type rat C6 glioma cells, but slightly synergistic in a CPT-resistant C6 cell line [44]. BT-474 human breast cancer cells which were utilized in this study were found to be inherently resistant to CPT, with a $\mathrm{D}_{50}$ of $100 \mu \mathrm{M}$, nearly 50 times that of CPT-resistant C6 cell lines reported in the literature [44]. However, when BT-474 cells were treated with CPT and DOX at a molar ratio of $\mathrm{R}=4.5$, the pair was able to inhibit $95 \%$ cell

growth at a CPT concentration 100-fold less than the $\mathrm{D}_{50}$ (Fig. 1a). Furthermore, this synergistic combination induced $>20 \%$ increase in pre-apoptotic cells compared to either single drug 
treatment (Fig. 2), thereby significantly improving efficacy at low drug concentrations. Hence, the finding of CPT and DOX synergy in CPT-resistant cells is consistent with previous studies, and suggests that CPT and DOX are most potent when used against highly CPT-resistant tumor cells.

In stark contrast, CPT and DOX exhibited extreme antagonism when exposed to bEnd.3 endothelial cells. The combination, regardless of ratio, actually reduced the toxicity of CPT or DOX, as indicated by improved endothelial cell viability (Fig. 1c). This vastly reduced combination activity in endothelial cells may be attributed to the inherently different cytotoxicity mechanisms CPT and DOX elicit in endothelial cells [41, 49, 50]. For example, DOX-mediated generation of hydrogen peroxide has been shown to play a pivotal role in endothelial cell apoptosis but not in tumor cell apoptosis [41]. Furthermore, top I enzymes present in endothelial cells exhibit higher resistance to CPT-induced oligonucleotide cleavage compared to those expressed in cancer cells $[49,50]$. Such differences in drug sensitivity may prevent synergistic interactions between CPT and DOX when exposed to endothelial cells, and provides a probable cause for reduction in combination activity. Because the synergistic interaction between DOX and CPT seems specific to tumor cells, the pair may be less toxic in vivo compared to the higher doses of free drug that are required to inhibit tumor growth.

The studies reported here also underscore the importance of drug ratios in determining potency. At $\mathrm{R}=0.1$, no improvement in cancer cell inhibition was seen (additive effect), but at $\mathrm{R}>2$, significant synergistic potency was apparent. This study verifies that precise drug ratios can govern combination synergy, as others have established $[32,33]$, and further emphasizes the need for controlled combination delivery to tumor tissue. Current combination administrations involve sequential intravenous infusions or bolus injections, and ignore the importance of drug ratios. Different drugs inherently undergo dissimilar pharmacokinetics, leading to local tumor drug ratios with inferior therapeutic effects. In the case of our particular chemotherapy combination, CPT exhibits a distribution half life of 71-90 minutes in humans [51], whereas that of DOX is much shorter, approximately 4 minutes [52]. Without inclusion of both drugs in a single vehicle, the pair will undergo uncoordinated pharmacokinetics, and the tumor will not be exposed to the optimal drug ratio which maximizes potency. This lack of unified drug pharmacokinetics may potentially explain little enhancements and even reductions of overall responses in clinical top I and II combinations.

In contrast to current clinical combination administrations, polymer-drug conjugates offer an advantageous method to deliver synergistic drug ratios to tumor tissue since precise drug concentrations can be incorporated through stoichiometric reactions. Conjugation of drugs to HA in particular is easily achieved through HA's native carboxylic acids present on the glucoronic acid monomers without prior modification of either polymer or drug [38, 53]. Carboxylic acids can react with nucleophilic moieties such as hydroxyls and free amines, functional groups which are present on many chemotherapy drugs. Specifically, aminolysis and esterification reactions were adapted for the conjugation of DOX and CPT to HA. Conjugation 
efficiencies of 1.6-1.8 mol\% DOX:HA and 1.6-5.9 mol\% CPT:HA were achieved with this method.

Most polymer-drug conjugates, including the clinical formulation of DOXhydroxyl(propyl methacrylamide) (HPMA), are synthesized via aminolysis, and result in similar conjugation efficiencies [54-56]. HA conjugation to drugs reduced their individual activity (Fig. 3), but did not compromise their synergistic effect, as seen in Fig. 7. The reduction of anticancer activity of HA-bound CPT or DOX compared to free drug may be attributed to the added barriers of drug release and endosomal escape prior to reaching intra-nuclear targets top I and top II. This shift in drug activity is more pronounced with DOX-HA compared to CPT-HA, and may be due to the presence of both of amide- and ester-bound DOX. In the case of CPT-HA, CPT is only conjugated via esterification. Slower hydrolysis of amides compared to esters may be the cause of reduced DOX release from the polymer (Fig. 5). The differential CPT and DOX release, however, does not compromise their synergy, as indicated by $\mathrm{CI}$ values less than 1 for $\mathrm{R}$ $>2$ in Fig. 6b. On the contrary, HA-conjugation may promote the pair's synergistic interactions against cancer cell proliferation. Whereas CPT and DOX in free solution only exhibit additive cancer cell kill at $\mathrm{R}=1$ (Fig. 1b), their HA-bound counterpart is synergistic (Fig. 6b). One hypothesis for this finding is that slower DOX release causes an effective free $\mathrm{R}$ greater than that bound to the polymer. Thus, the intracellular free combination released from the polymer results in $\mathrm{R}>2$, meeting the synergistic requirement for CPT and DOX. Free CPT and DOX are more synergistic at greater $\mathrm{R}$ values (Fig. 1b), with no apparent upper limit. In the case of polymerbound CPT and DOX, however, there appears to be an optimal polymer-bound R, as indicated by a greater $\mathrm{CI}$ for $\mathrm{R}=9$ compared to $\mathrm{R}=4.5$. Although this finding is not completely understood, one possible explanation is that a minimum DOX-HA concentration is required in order for the concentration of released DOX to be therapeutically active and synergistic with CPT. Because only $25 \%$ of DOX is released from DOX-HA over a period of 72 hours, exposure of cancer cells to larger HA-bound R can significantly reduce the concentration of free DOX exposed to cancer cells. It may be possible that a threshold DOX concentration is required for synergistic interactions with CPT. Further studies will be needed in order to verify whether threshold drug concentrations exist for synergistic drug interactions.

CPT and DOX simultaneously conjugated to $\mathrm{HA}$ in a ratio of $\mathrm{R}=3.2$ also synergistically inhibited cancer cell growth compared to CPT-HA or DOX-HA. When introduced intravenously, the incorporated drug ratios on the dual-drug-conjugated HA should maintain throughout circulation and at the tumor site; such carriers are deemed "ratiometric" and are hence projected to capture combination chemotherapy efficacy in vivo. Indeed, when CPT-HADOX was introduced into mice bearing $4 \mathrm{~T} 1$ tumors, a $50 \%$ reduction in tumor volume was achieved (Fig. 8a). Free drug solutions comprised of CPT and DOX in the same molar ratio $(\mathrm{R}=3.2)$ elicited slightly more therapeutic effect, with $70 \%$ tumor volume reduction relative to untreated mice. Histological analyses, however, show that CPT-HA-DOX is more effective at local potency (Fig. 8c-d), since it induces higher grade necrosis and apoptosis compared to free 
CPT + DOX. Chemotherapy drugs commonly initiate cancer cell death via apoptosis, but there are many potential benefits in necrotic-inducing agents $[57,58]$. In contrast to apoptosis, necrosis is a rapid cell death mechanism which does not require a complex cascade of biochemical events [58]. Necrosis is also indiscriminatory in that the affected cells need not be in a certain cycle phase or express certain genes, and once initiated, immune cells are recruited in an inflammatory response to further attack the tumor [57]. Hence, necrotic-inducing agents can result in more rapid and vigorous cancer cell death compared to apoptotic-inducing drugs. Free CPT+DOX attacks predominantly via apoptotic mechanisms, whereas CPT-HA-DOX exploits both potent mechanisms.

Of particular significance is the low doses utilized for these immense therapeutic effects, $2 \mathrm{mg} / \mathrm{kg} \mathrm{CPT}$ and $1.05 \mathrm{mg} / \mathrm{kg}$ DOX. Previous literature reports of murine tumor models treated with intravenous free drug solutions show that $2 \mathrm{mg} / \mathrm{kg}$ of free DOX by itself is incapable of significantly reducing tumor volume $[59,60]$, and that $1.5 \mathrm{mg} / \mathrm{kg}$ free CPT administered alone results in 50\% tumor reduction albeit with high variability [61]. In comparison, the studies reported here have identified a potent chemotherapy combination with high activity at low doses, and that HA-conjugation of synergistic ratios of CPT and DOX preserves the combination's potency in vivo.

Another challenge of course, in translating a powerful cytotoxic drug pair to a therapeutic, is to not just promote accumulation in tumor tissue, but to also prevent its exposure to healthy organs - a feat which can also be mediated with HA-conjugation. Here, the delivery vehicle itself targets tumor tissue, and additional targeting ligands are not needed. CD44, a transmembrane glycoprotein overexpressed in many cancer types and in low expression in other tissues, is a principal receptor of HA; thus, drug conjugation to HA can shift accumulation in tumors rather than healthy tissue [62-64]. The natural abundance of HA in the human body also renders it biocompatible and biodegradable. Negligible body weight changes (Fig. 8b) and H\&E analysis of essential organs (Fig. S7) suggest no toxicity or side effects in mice treated with CPT-HA-DOX, with the exception of mild inflammation in the heart. This may be explained by CD44 receptors and a large abundance of natural HA present in the heart [65]. Surprisingly, free solution CPT + DOX was also non-toxic to mice, with negligible body weight chances and no inflammation in the heart, lungs, liver, nor the spleen. Since CPT and DOX synergistically inhibit cancer cell growth for a wide range of ratios, the antitumor activity of this particular pair may not be severely impacted by the uncoordinated drug pharmacokinetics when administered as a free solution. Furthermore, the lack of chemotherapy toxicity may be attributed to the very low doses of CPT and DOX needed for synergistic tumor reduction. Thus, both HA-bound and free $\mathrm{CPT}+\mathrm{DOX}$ were well-tolerated formulations with compelling therapeutic efficacies.

While both CPT-HA-DOX and free CPT+DOX exhibited high therapeutic potential and minimal toxicity in mice, free solution combinations of top I and II inhibitors are notorious for inducing adverse side effects in humans. The most common repercussion of top I and II inhibitors, when administered as free drugs, is hematological toxicity [3-7, 9, 12, 14-16], and 
dose-limiting neutropenia has been reported to occur in as many as $50 \%$ of patients treated with top I and II poisons [13]. Polymer-conjugation to DOX, specifically DOX-HPMA, has already been shown to significantly reduce dose-limiting toxicities compared to free DOX [31], and is compelling evidence for the clinical use of polymer-bound drugs compared to free drugs. However, by identifying optimal synergistic ratios for tumor reduction, it may be possible to administer low, non-toxic free drug doses of the combination, as demonstrated here. Another advantage of polymer-drug conjugates is the preservation of synergistic drug ratios. Collectively, these findings suggest that CPT-HA-DOX has the potential of reducing lifethreatening toxicities and improving therapeutic efficacy compared to free CPT + DOX, although additional studies are required for further assessment, including an assessment of the dependence of efficacy on dosing frequency.

\section{Conclusion}

We report the discovery of optimal ratios of top I and II inhibitors CPT and DOX which synergistically inhibit cancer cell growth, and plausible non-toxic methods of their multi-drug delivery to tumors. Due to the potent synergy between CPT and DOX, only low doses of each drug are required to significantly reduce tumor volumes in murine 4T1 models - doses which elicit no visible toxicity in healthy organs. HA-conjugation serves as another clinically translatable method for CPT and DOX administration, with the additional advantages of preserved synergistic drug ratios from the site of injection to tumor tissue, and enhanced tumor accumulation. The latter method can be broadly applied to various combination chemotherapy methods, since the conjugation chemistries are not specific to just top I and II inhibitors, and can particularly be advantageous for combinations which elicit adverse effects in the free drug form. Both formulations, free drug and HA-bound combinations, achieved promising tumor reduction in mice and further clinical evaluation will shed light on the applicability of one formulation over the other.

\section{Acknowledgements}

The authors would like to acknowledge the use of the NRI-MCDB Microscopy Facility, funded by NIH Grant No. 1 S10 OD010610-01A1, as well as the Biological Nanostructures Laboratory within the California NanoSystems Institute, supported by the University of California, Santa Barbara and the University of California, Office of the President. A.C.A. was supported by the NSF Graduate Research Fellowship under Grant No. DGE-1144085 and also by UCSB's Center for Bioengineering under the Mellichamp Fellowship for Graduate Students, and D.R.V. was supported by NSF Graduate Research Fellowship under Grant No. DGE-1144085. We are also grateful to Dr. Mary Raven for her technical support with confocal microscopy, to Ronni Wynn and Dr. Manuel A. Garcia for help with in vivo studies, to Zoë Fuchs and Joshua Sanchez De Oliveira for assistance with experimental preparation, and to Dr. Louis Chin Jones for helpful discussions which have aided in directing the research. 


\section{References}

1. Devita Jr, V.T., R.C. Young, and G.P. Canellos, Combination versus single agent chemotherapy: a review of the basis for selection of drug treatment of cancer. Cancer, 1975. 35(1): p. 98-110.

2. Frei, E., E.J. Freireich, and E. Gehan, Studies of sequential and combination antimetabolite therapy in acute leukemia: 6-mercaptopurine and methotrexate. blood, 1961. 18: p. 431-454.

3. Garcia, A.A., et al., Phase I clinical trial of topotecan and pegylated liposomal doxorubicin. Cancer Invest, 2005. 23(8): p. 665-70.

4. Goff, L.W., et al., A phase I trial of irinotecan alternating with epirubicin in patients with advanced malignancies. Am J Clin Oncol, 2008. 31(5): p. 413-6.

5. Harada, T., et al., A phase I/II trial of irinotecan plus amrubicin supported with G-CSF for extended small-cell lung cancer. Jpn J Clin Oncol, 2014. 44(2): p. 127-33.

6. Hotta, K., et al., Phase I study of irinotecan and amrubicin in patients with advanced non-small-cell lung cancer. Anticancer Res, 2005. 25(3c): p. 2429-34.

7. Lau, D., et al., Population-based phase I trial of irinotecan and epirubicin. Am J Clin Oncol, 2008. 31(3): p. 226-30.

8. Mirchandani, D., et al., Phase I study of combined pegylated liposomal doxorubicin with protracted daily topotecan for ovarian cancer. Clinical cancer research : an official journal of the American Association for Cancer Research, 2005. 11(16): p. 5912-9.

9. Morgensztern, D., et al., A phase I study of pegylated liposomal doxorubicin and irinotecan in patients with solid tumors. Chemotherapy, 2009. 55(6): p. 441-5.

10. Nishimura, S., et al., Phase II study of irinotecan plus doxorubicin for early recurrent or platinum-refractory ovarian cancer: interim analysis. International journal of gynecological cancer : official journal of the International Gynecological Cancer Society, 2007. 17(1): p. 159-63.

11. Nogami, N., et al., Phase II study of irinotecan and amrubicin in patients with relapsed non-small cell lung cancer: Okayama Lung Cancer Study Group Trial 0402. Acta oncologica (Stockholm, Sweden), 2012. 51(6): p. 768-73.

12. Oshita, F., H. Saito, and K. Yamada, Dose escalation study of amrubicin in combination with fixed-dose irinotecan in patients with extensive small-cell lung cancer. Oncology, 2008. 74(1-2): p. 7-11.

13. Ryan, C.W., et al., A phase I study of liposomal doxorubicin (Doxil) with topotecan. Am J Clin Oncol, 2000. 23(3): p. 297-300.

14. Shoji, T., et al., A phase I study of irinotecan and pegylated liposomal doxorubicin in recurrent ovarian cancer (Tohoku Gynecologic Cancer Unit 104 study). Cancer Chemother Pharmacol, 2014. 73(5): p. 895-901.

15. Smith, S.M., et al., Sequential doxorubicin and topotecan in relapsed/refractory aggressive non-Hodgkin's lymphoma: results of CALGB 59906. Leuk Lymphoma, 2006. 47(8): p. 1511-7.

16. Wagner, S., et al., Pegylated-liposomal doxorubicin and oral topotecan in eight children with relapsed high-grade malignant brain tumors. J Neurooncol, 2008. 86(2): p. 175-81.

17. Xenidis, N., et al., Alpha multicenter phase II study of pegylated liposomal doxorubicin in combination with irinotecan as second-line treatment of patients with refractory smallcell lung cancer. Cancer Chemother Pharmacol, 2011. 68(1): p. 63-8. 
18. Sugimoto, Y., et al., Elevated expression of DNA topoisomerase II in camptothecinresistant human tumor cell lines. Cancer Res, 1990. 50(24): p. 7962-5.

19. Oguro, M., et al., Collateral drug sensitivity induced in CPT-11 (a novel derivative of camptothecin)-resistant cell lines. Biomed Pharmacother, 1990. 44(4): p. 209-16.

20. Krauze, M.T., et al., Convection-enhanced delivery of nanoliposomal CPT-11 (irinotecan) and PEGylated liposomal doxorubicin (Doxil) in rodent intracranial brain tumor xenografts. Neuro Oncol, 2007. 9(4): p. 393-403.

21. Shaikh, I.M., et al., Liposome co-encapsulation of synergistic combination of irinotecan and doxorubicin for the treatment of intraperitoneally grown ovarian tumor xenograft. $\mathrm{J}$ Control Release, 2013. 172(3): p. 852-861.

22. Hanada, M., T. Noguchi, and T. Yamaoka, Amrubicin, a novel 9-aminoanthracycline, enhances the antitumor activity of chemotherapeutic agents against human cancer cells in vitro and in vivo. Cancer Sci, 2007. 98(3): p. 447-54.

23. Takigawa, N., et al., The combination effect of amrubicin with cisplatin or irinotecan for small-cell lung cancer cells. Oncol Rep, 2006. 15(4): p. 837-42.

24. Barua, S. and S. Mitragotri, Synergistic targeting of cell membrane, cytoplasm, and nucleus of cancer cells using rod-shaped nanoparticles. ACS Nano, 2013. 7(11): p. 955870.

25. Grant, S.C., et al., Single-agent chemotherapy trials in small-cell lung cancer, 1970 to 1990: the case for studies in previously treated patients. J Clin Oncol, 1992. 10(3): p. 484-98.

26. Lammers, T., et al., Effect of physicochemical modification on the biodistribution and tumor accumulation of HPMA copolymers. J Control Release, 2005. 110(1): p. 103-18.

27. Sadekar, S., et al., Comparative biodistribution of PAMAM dendrimers and HPMA copolymers in ovarian-tumor-bearing mice. Biomacromolecules, 2011. 12(1): p. 88-96.

28. Tabata, Y., Y. Murakami, and Y. Ikada, Tumor accumulation of poly(vinyl alcohol) of different sizes after intravenous injection. J Control Release, 1998. 50(1-3): p. 123-33.

29. Kopecek, J., et al., Water soluble polymers in tumor targeted delivery. J Control Release, 2001. 74(1-3): p. 147-58.

30. Duncan, R., Development of HPMA copolymer-anticancer conjugates: clinical experience and lessons learnt. Adv Drug Deliv Rev, 2009. 61(13): p. 1131-48.

31. Vasey, P.A., et al., Phase I clinical and pharmacokinetic study of PK1 [N-(2hydroxypropyl)methacrylamide copolymer doxorubicin]: first member of a new class of chemotherapeutic agents-drug-polymer conjugates. Cancer Research Campaign Phase I/II Committee. Clin Cancer Res, 1999. 5(1): p. 83-94.

32. Tardi, P., et al., In vivo maintenance of synergistic cytarabine:daunorubicin ratios greatly enhances therapeutic efficacy. Leuk Res, 2009. 33(1): p. 129-39.

33. Mayer, L.D., et al., Ratiometric dosing of anticancer drug combinations: controlling drug ratios after systemic administration regulates therapeutic activity in tumor-bearing mice. Mol Cancer Ther, 2006. 5(7): p. 1854-63.

34. Platt, V.M. and F.C. Szoka, Jr., Anticancer therapeutics: targeting macromolecules and nanocarriers to hyaluronan or CD44, a hyaluronan receptor. Mol Pharm, 2008. 5(4): p. 474-86.

35. Journo-Gershfeld, G., et al., Hyaluronan oligomers-HPMA copolymer conjugates for targeting paclitaxel to CD44-overexpressing ovarian carcinoma. Pharm Res, 2012. 29(4): p. 1121-33. 
36. Choi, K.Y., et al., Self-assembled hyaluronic acid nanoparticles for active tumor targeting. Biomaterials, 2010. 31(1): p. 106-14.

37. Chou, T.C., Theoretical basis, experimental design, and computerized simulation of synergism and antagonism in drug combination studies. Pharmacol Rev, 2006. 58(3): p. 621-81.

38. Lee, H., K. Lee, and T.G. Park, Hyaluronic acid-paclitaxel conjugate micelles: synthesis, characterization, and antitumor activity. Bioconjug Chem, 2008. 19(6): p. 1319-25.

39. Minko, T., P. Kopeckova, and J. Kopecek, Efficacy of the chemotherapeutic action of HPMA copolymer-bound doxorubicin in a solid tumor model of ovarian carcinoma. Int $\mathbf{J}$ Cancer, 2000. 86(1): p. 108-17.

40. Walton, M.I., et al., Constitutive expression of human Bcl-2 modulates nitrogen mustard and camptothecin induced apoptosis. Cancer Res, 1993. 53(8): p. 1853-61.

41. Wang, S., et al., Doxorubicin induces apoptosis in normal and tumor cells via distinctly different mechanisms. intermediacy of $\mathrm{H}(2) \mathrm{O}(2)$ - and p53-dependent pathways. J Biol Chem, 2004. 279(24): p. 25535-43.

42. Pulaski, B.A. and S. Ostrand-Rosenberg, Mouse $4 T 1$ breast tumor model. Curr Protoc Immunol, 2001. Chapter 20(2): p. Unit 202.

43. Fantozzi, A. and G. Christofori, Mouse models of breast cancer metastasis. Breast Cancer Res, 2006. 8(4): p. 212.

44. Pavillard, V., et al., Effects of the combination of camptothecin and doxorubicin or etoposide on rat glioma cells and camptothecin-resistant variants. Br J Cancer, 2001. 85(7): p. 1077-83.

45. Budman, D.R. and A. Calabro, In vitro search for synergy and antagonism: evaluation of docetaxel combinations in breast cancer cell lines. Breast Cancer Res Treat, 2002. 74(1): p. 41-6.

46. Budman, D.R. and A. Calabro, Zoledronic acid (Zometa) enhances the cytotoxic effect of gemcitabine and fluvastatin: in vitro isobologram studies with conventional and nonconventional cytotoxic agents. Oncology, 2006. 70(2): p. 147-53.

47. Budman, D.R., A. Calabro, and W. Kreis, In vitro evaluation of synergism or antagonism with combinations of new cytotoxic agents. Anticancer Drugs, 1998. 9(8): p. 697-702.

48. Liu, Y., et al., Codelivery of doxorubicin and paclitaxel by cross-linked multilamellar liposome enables synergistic antitumor activity. Mol Pharm, 2014. 11(5): p. 1651-61.

49. Clements, M.K., S. Wasi, and S.S. Daoud, Camptothecin exhibits selective cytotoxicity towards human breast carcinoma as compared to normal bovine endothelial cells in vitro. Anti-cancer drugs, 1996. 7(8): p. 851-857.

50. Jones, C.B., et al., Sensitivity to camptothecin of human breast carcinoma and normal endothelial cells. Cancer chemotherapy and pharmacology, 1997. 40(6): p. 475-483.

51. Gottlieb, J.A., et al., Preliminary pharmacologic and clinical evaluation of camptothecin sodium (NSC-100880). Cancer Chemother Rep, 1970. 54(6): p. 461-70.

52. Gabizon, A., et al., Prolonged circulation time and enhanced accumulation in malignant exudates of doxorubicin encapsulated in polyethylene-glycol coated liposomes. Cancer Res, 1994. 54(4): p. 987-92.

53. Luo, Y. and G.D. Prestwich, Hyaluronic acid-N-hydroxysuccinimide: a useful intermediate for bioconjugation. Bioconjug Chem, 2001. 12(6): p. 1085-8.

54. Dvorak, M., P. Kopeckova, and J. Kopecek, High-molecular weight HPMA copolymeradriamycin conjugates. J Control Release, 1999. 60(2-3): p. 321-32. 
55. Lammers, T., et al., Simultaneous delivery of doxorubicin and gemcitabine to tumors in vivo using prototypic polymeric drug carriers. Biomaterials, 2009. 30(20): p. 3466-75.

56. Shiah, J.J., et al., Biodistribution of free and N-(2-hydroxypropyl)methacrylamide copolymer-bound mesochlorin e(6) and adriamycin in nude mice bearing human ovarian carcinoma OVCAR-3 xenografts. J Control Release, 1999. 61(1-2): p. 145-57.

57. Amaravadi, R.K. and C.B. Thompson, The roles of therapy-induced autophagy and necrosis in cancer treatment. Clinical Cancer Research, 2007. 13(24): p. 7271-7279.

58. Kiaris, H. and A.V. Schally, Apoptosis versus necrosis: which should be the aim of cancer therapy? Experimental Biology and Medicine, 1999. 221(2): p. 87-88.

59. Ottewell, P.D., et al., Antitumor effects of doxorubicin followed by zoledronic acid in a mouse model of breast cancer. Journal of the National Cancer Institute, 2008. 100(16): p. 1167-1178.

60. Chen, Y., et al., Multifunctional nanoparticles delivering small interfering RNA and doxorubicin overcome drug resistance in cancer. J Biol Chem, 2010. 285(29): p. 2263950 .

61. Watanabe, M., et al., In vivo antitumor activity of camptothecin incorporated in liposomes formulated with an artificial lipid and human serum albumin. J Control Release, 2008. 127(3): p. 231-8.

62. Bourguignon, L.Y., Hyaluronan-mediated CD44 activation of RhoGTPase signaling and cytoskeleton function promotes tumor progression. Semin Cancer Biol, 2008. 18(4): p. 251-9.

63. Mero, A. and M. Campisi, Hyaluronic Acid Bioconjugates for the Delivery of Bioactive Molecules. Polymers, 2014. 6(2): p. 346-369.

64. Mackay, C.R., et al., Expression and modulation of CD44 variant isoforms in humans. $\mathrm{J}$ Cell Biol, 1994. 124(1-2): p. 71-82.

65. Hellstrom, M., B. Johansson, and A. Engstrom-Laurent, Hyaluronan and its receptor CD44 in the heart of newborn and adult rats. Anat Rec A Discov Mol Cell Evol Biol, 2006. 288(6): p. 587-92. 


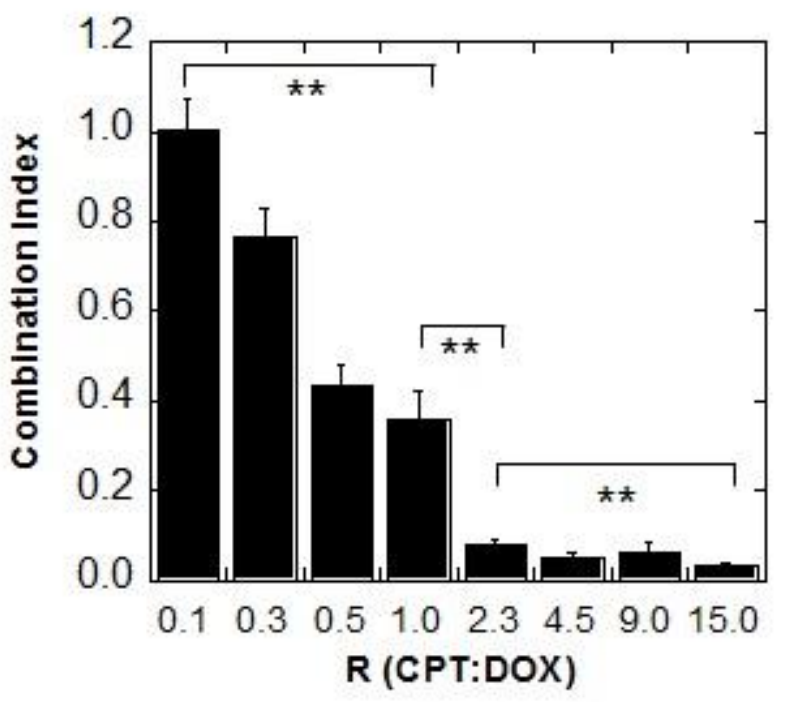

Graphical abstract 